\title{
Research and Development of Online Teaching Platform for Mobile Internet
}

\author{
Yujiao WANG ${ }^{1, a}$, Haiyun LIN $^{1, *}$, Peiping ZHU ${ }^{1, b}$ \\ ${ }^{1}$ Department of Physical Science and Technology, Kunming University Yunnan, Kunming 650214, \\ China \\ aemail:tjwyj817@163.com, *e-mail:Ihy198@163.com, bemail: 57056253@qq.com
}

Keywords: Mobile Internet, Structure of Platform, Test Method, Performance Test

\begin{abstract}
Through the analysis on the characteristics and demands of the online teaching platform, the web-based teaching platform based on the mobile internet is established. Analysis and test on the performance of the online teaching platform has been carried out. The test result shows that this platform can realize the flexible expansion and meet the function and usage requirement of the open online teaching according to users demands.
\end{abstract}

\section{Introduction}

The universal application of the mobile internet, especially the development of the big data, cloud computing, mobile internet, etc, is profoundly changing the education status, pushing forward the education digitization, networking and intelligence [1]. From the viewpoint of the education to view the mobile internet, it is easy to find that this tide not only brings about the reform of the education technology, but also brings about impact on the study, teaching, and organizational mode, and deep influence on the education concept and system. Due to the current requirement for the balance development of the education resource and the strong advocating for the sharing, the demands for the online teaching and study have been rapidly increased. To solve the education from closing to opening has important significance for the supervisors of the Ministry of Education and the usage units. The organic combination of the mobile internet and online teaching platform makes the education management more efficient and simpler [2]. The integration of the activities and resource after class and the traditional teaching constructs the learning activities suitable for the students to the greatest extent, which improves the education quality.

\section{Mobile internet technology}

\subsection{Overview of the mobile internet}

Mobile Internet (MI) is a new business that adopting the mobile radio communication method to achieve the business and service through the intelligent mobile terminal, including the terminal, software and application. The terminal layer includes the intelligent smart phone, tablet PC, e-book, MID, etc. The software includes operation system, middleware, database, security software, etc. The application layer includes different application and service, such as leisure recreation, tool and media, finance, etc.

\subsection{Mobile internet structure}

From the layers, the mobile internet can divided into terminal/equipment layer, access/network layer and application/business layer. The most obvious feature is diversity. The application and business types are various. The corresponding communication mode and service quality requirement is different. The access layer supports many kinds of wireless access mode. However, in the network layer, IP agreement is focused. The terminal is also diversified. It attaches great importance to the individualization and intelligence. On a terminal, several applications are operated. WWRF holds that the internet is self-adapting, individualized and environment perceivable service. The mobile network reference mode it provides is shown in below photo. Various applications achieve the user interaction support or mobile middle part support through the 
open application interface. The mobile middle part is composed of several universal service elements, including modeling service, existence service, mobile data management, configuration management, service discovery, event notice, environment monitoring, etc. The internet protocol mainly has IP service agreement, transport protocols, mechanism protocol, networking protocol, control and management protocol, etc. Meanwhile, it takes charge of the adaptive function from the network layer to the link layer. The operation system completes the interaction between the upper layer protocol and lower hardware resource. The hardware/firmware refers to the device constituting the terminal and equipment.

\section{Online education platform framework}

The teaching management system integrates the information. On the framework layer of the education application system, it gets involved in many dispersed, internal and external information processing process, and provides integration bond to the education, tries to integrate framework, different development platform and different application database and different operation system with the minimum cost based on the education information of the platform. Based on the three layers of ASP.NET, the online education platform is divided into data storage interface layer, application layer, information integration access layer and portal.

Framework division: Application layer is the object that the education information integration platform should integrate, including the structural and non-structural data from different system, including the internal or external application systems of the education. It refers all information resource that already exists. Information integration access layer. It realizes the uniform access of the information, provides the uniform management and authorization of all information resource, and provides bottom layer support for the different types of information integration, including the local and remote application integration components, providing bottom layer component base for the education information integration platform to realize the resource integration [3]. Display layer. Only need to click and log in the portal, and then achieve the focused information. The uniform view management user can set own individual output, only focus on how to display the information, and display the integrated information resource to the user's outermost layer and information integration platform.

The network education platform is displayed by the education portal to the users. Different integration components are adopted for processing, and establishing the corresponding relationship of user password transformation between different systems, transmitting to front-end display layer. In this framework, through the resource definition and distribution, according to the information resource type from the after-end application layer, the integration components and all information application in the information integration access layer are regarded as the after-end application layer.

\section{Performance Test Method of Platform}

Target of Platform performance is to demonstrate the performance of Platform service under load condition. The best method of carrying out performance test is to enable many test customers to use complete service test, including request submission and answer demonstration [4]. Performance test dose not only monitors response rate of server by designated concurrent request number, but also needs to test each kind of load whether it can cause function fault of cloud service. Therefore, it requires performance test of service can all set or make performance test scene (it mainly means designated load grade, load distribution etc) to carry out test suite of performance. According to requirement, the test except to emphasize designated efficiency and performance indexes such as concurrent customer number, response time, source utilization condition, it also needs to carry out evaluation for efficiency index such as speed-up ratio of response time (demonstrate additional expenses, it is used for concurrent communication ability), command efficiency etc [5].

\subsection{Speed-up ratio}

Speed-up ratio of parallel operation 
Measurement method: $S(q)=\frac{T s}{T_{p}(q)}$

Ts is implementation time of sequential algorithm, $T_{p}(q)$ is the time of processor parallel implementation by using $q$.

Speed-up ratio of response time:

Measurement method: $Y=\left(T_{B}-T_{A}\right) / T_{A}$

$T_{A}$ is the total implementation response time of sequential algorithm, $T_{B}$ is the response time of single processor implementation by using many processors.

\subsection{Command efficiency}

Measurement method: $E=n / t$

$n$ indicates the command number implemented in time of $t, t$ means the time of implementing $n$ pieces of command, the million times of implementing command in second, test index is directly generated by test procedure [7].

\subsection{WEB Service}

Average distribution of access

Measurement method: $C=u \times L / T$

$C$ is the average concurrent users, $u$ is the registration users, $L$ is the average time for use registration, $T$ means the time length of visit.

\subsection{Access integration}

Measurement method: $C=(n \times 80 \%) \times L /(T \times 80 \%)$

$\mathrm{C}$ is the average concurrent user data, $n$ is the number of registered users, $L$ is the average time length of registration for each user,

\subsection{Peak value of concurrent users}

Measurement method: $\widehat{C}=C+3 \sqrt{C}$ Of which, $C$ is the number of average concurrent users

Element name of measurement: extendibility estimation of system

Measurement method: $C^{\prime}=C \times(1+m)^{t}$

$C^{\prime}$ is the number of expected concurrent users, $C$ is the number of average concurrent users, $m$ is the growth of business in unit time, $t$ is the number of calculating expected time unit.

\subsection{Average response delay}

Measurement method: $M R D=\operatorname{Sum} / N$

$$
\text { Sum }=\sum_{i=1}^{N} L i
$$

Of which, MRD means average response delay, Sum means the time sum of users sending out and receiving its response, $\mathrm{Li}$ means the time of users sending request $i$ and receiving its response, $N$ means the number sum of system processing request.

\subsection{Relative mistake group load}

Measurement method:

$$
p=\sum_{i=1}^{k}\left(B_{i}-B_{a v e}\right)
$$

Of which, $B_{i}$ means the load of point ${ }^{i}, B_{\text {aue }}$ means the average load of $k$ points, $k$ load of calculating point ${ }^{k}$,

$B_{k}=U_{p} \cdot C$, of which, $U_{p}$ means the CPU usage ratio of point $k, C$ means the consecutive numbers of point $k$. 


\section{System test method}

Regard the test object as an open box, white box test. The online teaching management system test adopts the white box test method. This test method applies the internal processing and result analysis method of the software to analyze the dynamic status of the system. In the software testing process, it completes the following work

According to the use case test of the software system, test user authentication module, analyze the searching and adding function of the local police station teaching data, carry out main function user case test aiming at the online teaching management system.

Under the designed network system framework, operate the software module under various scenes, analyze and summarize the basic parameter of the software operation according to the parameters displayed on the test tool, study the performance effect, test the CPU usage ratio of the system and internal storage, and response time on average . The test result is shown in the Fig.1.

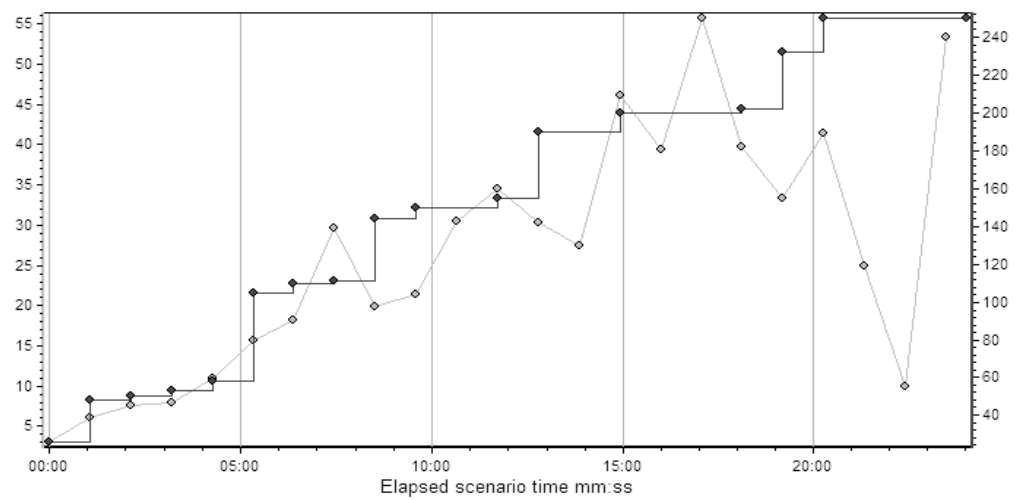

Fig 1 Analysis chart of the affair response and maximum concurrent users

\section{Test result analysis}

The test result shows that, through various conditions, the system operation scene operates the mobile network platform and tests the operated online teaching management system. The embodied performance parameters achieve the operation response curve of each module and analyze the page of the system through the curve. The response time meets the actual online teaching management requirement. Meanwhile, during the visiting, different number of users tests the user log-in validation, information adding, information searching, and user case through the collected operation data. It displays the online teaching management system, and the user case function of each item, which meets the design requirements.

Regarding the performance test, after detailed testing, it shows that CPU usage rate, internal storage usage rate, and average access time of the online teaching management system can meet the system index requirements. The maximum number of the concurrent users is 2000, which basically can meet the requirements of the open online teaching management under the mobile internet.

\section{Conclusion}

The rapid development of the mobile internet brings opportunities and challenges to the current society and education. It has profound influence on the education resource, education institute, learning modes and teaching modes, etc, which changes the education concept. Such change fundamentally affect the teaching modes of the schools, so the teachers should constantly improve and innovate their teaching methods, integrate various superior education and teaching resource, establish education resource network platforms, which is favorable for promoting the reform of the education concept, and making the students become the subject of the study. To solve the closure of the education resource, we put forward development proposals for the education platform of the mobile internet, which can fundamentally solve the problems about the education resource sharing and muti-media interaction. 


\section{References}

[1] H Lin, Y Wang, J Cai, C Li, Design and development of education cloud platform,Journal of Chemical \& Pharmaceutical Research, vol.6, pp.383-386, 2014.

[2] Zhan Qinglong, Yang Mengjia, Research on Maker Education 2.0 and Smart Learning Activities from the Perspective of “Internet Plus”, Journal of Distance Education,vol.6, pp.24-31, 2015.

[3] Li Jian, Construction and application of teaching system based on mobile network,Journal of Liaoning Institute of Science and Technology,vol.17, pp.26-29, 2015.

[4] HY Lin, YJ Wang, The Research of M-Learning Platform Based on 4G Network,Advanced Materials Research,vol.989-994,pp.4157-4160,2014.

2014,

[5] Xin LI, Zhanhuai LI, Xiao Zhang,” Massive Storage Oriented File System Evaluation Benchmark”, Computer Engineering, 2011(15), pp.55-57. 